\title{
Pollution studies for high order Isogeometric Analysis and Finite Element for acoustic problems
}

\author{
Ganesh C. Diwan ${ }^{1, *}, \quad$ M Shadi Mohamed ${ }^{2}$ \\ ${ }^{1}$ Department of Medical Physics and Biomedical Engineering, University College London, UK \\ ${ }^{2}$ School of Energy, Geoscience, Infrastructure and Society, Heriot-Watt University, Edinburgh, UK
}

\begin{abstract}
It is well known that Galerkin finite element methods suffer from pollution error when solving wave problems. To reduce the pollution impact on the solution different approaches were proposed to enrich the finite element method with wave-like functions so that the exact wavenumber is incorporated into the finite element approximation space. Solving wave problems with isogeometric analysis was also investigated in the literature where the superior behaviour of isogeometric analysis due to higher continuity in the underlying basis has been studied. Recently, a plane wave enriched isogeometric analysis was introduced for acoustic problems. However, it remains unquantified the impact of these different approaches on the pollution or how they perform compared to each other. In this work, we show that isogeometric analysis outperforms finite element method in dealing with pollution. We observe similar behaviour when both the methods are enriched with plane waves. Using higher order polynomials with fewer enrichment functions seems to improve the pollution compared to lower order polynomials with more functions. However, the latter still leads to smaller errors using similar number of degrees of freedom. In conclusion, we propose that partition of unity isogeometric analysis can be an efficient tool for wave problems as enrichment eliminates the need for domain re-meshing at higher frequencies and also due to its ability to capture the exact geometry even on coarse meshes as well as its improved pollution behaviour.
\end{abstract}

Keywords. Pollution error; Finite element method; Isogeometric analysis; Partition of unity; Wave problems; Wave scattering, Helmholtz equation.

\section{Introduction}

In this paper we look into solving the time harmonic wave problem using finite element methods. The problem is highly relevant to applications in acoustics and electromagnetics and often involves solving the Helmholtz equation. Finite element method (FEM) is chosen here due to its flexibility in handling complex geometries. It is well known that the standard FEM with linear or quadratic basis functions is limited to low wavenumbers/long wavelengths and requires, as a rule of thumb, around ten degrees of freedom per wavelength in order to achieve engineering accuracy. However,

\footnotetext{
${ }^{*}$ Corresponding author: g.diwan@ucl.ac.uk
} 
theory and numerical studies indicate that a constant resolution rule is not sufficient to maintain a certain accuracy as wavenumber increases and the method severely suffers from pollution as the wavenumber increases [1]. Thus, for domains that are relatively large in terms of the wavelength, highly refined meshes become necessary. In such cases the total number of degrees of freedom can be prohibitively large. Especially, for three-dimensional problems if the domain is large enough to accommodate more than few thousand wavelengths per-direction then FEM becomes impractical even with high performance computing.

The pollution can be understood as the gap between the errors of Galerkin solution and the best approximation that is possible with the underlying Galerkin basis. Because the FEM solution is dispersive, the pollution can also be understood as the discrepancy between problem wavenumber and the wavenumber recovered with the finite element method [1, 2]. It is well established that pollution can be overcome (i.e. where the gap between FEM and best approximation becomes negligible) only with sufficiently refined meshes. The theoretical and computational studies indicate the quasi-optimality of the method can be reached in the asymptotic range and that this depends on the wavenumber and the approximation order. For example see [1, 3,6] where the pollution is extensively studied and the error estimates are provided in terms of the element size and polynomial order. Specifically, Melenk and Sauter [5,6] prove that FEM is quasi-optimal when $k^{p+1} h^{p}<C$, with $k$ as the wavenumber, $h$ as the effective meshwidth and $p$ as the polynomial order. Thus, although the pollution still affects high order methods, its effect can be significantly reduced by increasing p. Ihlenburg and Babuška provide 'pre-asymptotic' error estimates in their first series of analysis papers [1,7]. Their numerical studies on the $h$-version of FEM indicate, that the FEM solution enters the asymptotic range only if $k^{2} h=C$ whereas for $k h=C$ the FE error cannot be controlled. A condition such as $k^{2} h=C$ can lead to large linear systems that are expensive to solve. Rarely in engineering practice, one is interested in solutions that match the best approximation. In fact for an engineering analyst, it is acceptable if the errors from FEM can be bounded within a desired accuracy even if the solution does not match to the best approximation.

In order to reduce the constant resolution requirement different researchers worked on improving the approximation properties of FEM. A major improvement came from creating new elements with oscillatory test functions. These elements enable achieving engineering accuracy on coarse meshes where each element is multiple times larger than the wavelength. Thus, a significant reduction in the computational efforts becomes possible. An early example of this enrichment based approach can be found in the work of Melenk and Babuška on the partition of unity method [8,9]. Their work proposes incorporating analytical solutions of the Helmholtz equation into the finite element space using the partition of unity property. This idea was later developed by Laghrouche et al. to recover scattered waves in two- and three-dimensions for acoustic [10,11] and elastic wave problems [12] using the partition of unity finite element method (PUFEM). Strouboulis et al. [13] studied the computational aspect of PUFEM where they considered multiple polynomial orders with plane wave enrichment. However, they conclude the pollution error cannot be completely eliminated but can be controlled with judicial choice of polynomial order and the number of plane waves. It is important to mention here that the round off errors can give slightly misleading results at high orders where the best approximation appears less accurate than (or very close to) FEM, see Tables 2 and 3 from [13 and also in [14]. Different aspects of the approach such as the enrichment functions, the convergence and the integration were studied in details by several authors including but not limited to [15 18].

The partition of unity approach uses continuous finite elements while other enrichment approaches often rely on discontinuous elements. Farhat et al. proposed the discontinuous enrichment method where plane waves or other oscillatory functions were used for the enrichment [19 21]. Another discontinuous approach is the ultraweak variational formulation 22 24]. Several other enrichment 
approaches based on discontinuous Galerkin elements are also available in the literature [25 27]. A recent literature overview on using enriched elements for solving the Helmholtz equation can be found in [28].

Despite the flexibility of FEM in dealing with the problem geometry, the exact representation of curved edges or surfaces can be a real challenge. In general, having small discrepancies between the exact and the discretized geometries can be tolerated. However, for wave problems small discrepancies can be a serious issue where small inaccuracies can significantly increase the solution error [16, 29]. Refining the finite element mesh close to curved edges is a standard procedure to reduce the error of the geometry representation. But often the resulting graded meshes can defy the efficiency gained through the enrichment. The decreased efficiency is caused mainly by the increased number of small elements. Moreover, the linear system of equation resulting form FEM discretization can become ill-conditioned if such graded meshes are enriched with a uniform number of enrichment functions 30$]$.

To have an exact geometry representation and to simplify the meshing process Hughes et al. [31] proposed the idea of isogeometric analysis (IGA). The key is to approximate the solution with FEM by using the same basis used to build the computer aided design (CAD) geometry. To this end the non-uniform rational B-splines (NURBS) that define the geometry are used to construct the basis functions for FEM. Hughes et al. have been able to show several advantages for the IGA approach including improvement in the accuracy for the same number of degrees of freedom compared to the standard FEM. This has motivated many research groups to adopt the new IGA for a wide range of FEM applications relevant to fluid-structure interaction [32, 33], electromagnetics [34, 35] structures [36,37] and acoustics [38]. More recently IGA was combined with the enrichment approach in fracture mechanics [39,40]. An overview of IGA and different related implementation aspects can be found in [41]. Using enriched IGA for solving the Helmholtz equation was first achieved with the boundary element method in two-dimensions [42] and then in three-dimensions [43]. More recently enriched finite elements were also developed for IGA 29]. The combination termed as the partition of unity isogeometric analysis (PUIGA) can circumvent the need for graded meshes, hence, eliminate all associated inefficiencies. Furthermore, the higher order basis used can also reduce the pollution error.

Although the pollution effect has been extensively studied for FEM and PUFEM, it is still unquantified what impact using IGA, not to mention PUIGA, has on it. In fact it was previously argued that IGA can eliminate any pollution [38]. In this paper we show that although the pollution error is significantly reduced with IGA, it is not completely eliminated even with higher order basis functions. We quantify the pollution effect on IGA with enriched and non-enriched elements by providing empirical estimates on the quasi-optimality constants. Comparisons to the standard polynomial FEM as well as PUFEM are carried out. Then the advantage of PUIGA is investigated by analysing the interior acoustics of a standard passenger car. The relatively complicated geometry of the vehicle makes it an ideal candidate for using NURBS. The same NURBS surfaces/patches describing the geometry, obtained from CAD software, are also used as a mesh for IGA. Thus, the often non-trivial computational costs associated with the FEM meshing/re-meshing process are reduced. This major advantage of IGA is usually lost in solving wave problems where it becomes necessary to refine the mesh whenever a higher frequency is considered. In this paper we show it is possible to retain this advantage using PUIGA. We recover the noise patterns in the car interior at frequencies for the audible range 0.5 to $20 \mathrm{kHz}$. This is achieved on a fixed coarse mesh by merely injecting an increased number of enrichment functions. The convergence is ensured by following the so called $q$-refinement approach. 
Following the introduction, we present the variational formulation of the considered problem. We then introduce the finite element discretization with Lagrangian and NURBS basis functions and the plane wave enrichment. Next, numerical experiments are run to investigate the pollution error associated with enriched and non-enriched basis functions. The PUIGA is then used for analysing the interior noise of a passenger car. We finish with concluding remarks.

\section{Variational formulation of the problem}

Consider $\Omega$ to be an open bounded domain in $\mathbb{R}^{2}$ where $\partial \Omega$ is the domain boundary. We consider the time harmonic wave problem where the scalar acoustic potential, $u$ is governed by the Helmholtz equation in $\Omega$ subject to some boundary conditions: find $u \in H^{1}(\Omega)$ such that

$$
\begin{aligned}
& \Delta u+k^{2} u=0, \quad \text { in } \quad \Omega \\
& \frac{\partial u}{\partial \mathbf{n}}+\mathrm{i} k u=g \quad \text { on } \quad \partial \Omega,
\end{aligned}
$$

where $g$ is a square integrable function, $k>0$ the wavenumber, $\Delta$ the Laplace operator, $\mathrm{i}=\sqrt{-1}$, and $\mathbf{n}$ the outward unit normal vector to the domain boundary. It should be stressed that $\mathbf{a}$ full exterior scattering problem is not considered in this work to avoid numerical errors from approximating radiation boundary conditions. Multiplying equation (1a) by a test function $v \in H^{1}(\Omega)$ and integrating by parts, we obtain the weak form: given a square integrable function $g$, find $u \in H^{1}(\Omega)$, s.t.

$$
\int_{\Omega}\left(\nabla u \overline{\nabla v}-k^{2} u \bar{v}\right) d \Omega+\mathrm{i} k \int_{\Gamma} u \bar{v} d \Gamma \quad=\int_{\Gamma} \bar{v} g d \Gamma
$$

with $H^{1}(\Omega)$ being the standard Sobolev space. The domain boundary are made up of nonintersecting parts where we consider a Robin type boundary condition. To solve the weak formulation (2) we define $\mathcal{T}_{h}$ to be a set of decompositions dividing $\Omega$ into non-overlapping and non self-intersecting elements where $h$ is the diameter of any $E \in \mathcal{T}_{h}$. Let $p$ be the given polynomial order of the method. We then have a finite dimensional discrete space, $V_{h} \subset H^{1}(\Omega)$ defined as:

$$
V_{h}=\left\{v \in H^{1}:\left.v\right|_{E} \in \mathbb{P}_{p}(E) \text { for each element } E \in \mathcal{T}_{h}\right\}
$$

where, $\mathbb{P}_{p}(E)$ is the space of polynomials defined on element $E$ and of order $p$. The discrete Galerkin form is then given by: find $u_{h} \in V_{h}$, s.t.

$$
\int_{\Omega}\left(\nabla u_{h} \overline{\nabla v_{h}}-k^{2} u_{h} \overline{v_{h}}\right) d \Omega+\mathrm{i} k \int_{\Gamma} u_{h} \overline{v_{h}} d \Gamma=\int_{\Gamma} \overline{v_{h}} g d \Gamma
$$

\section{Finite Element Approximation}

Let $V_{h}$ contains a linear combination of 'basis functions' $\mathcal{N}_{A}$, with, $A=1, \cdots, N$, so that,

$$
u_{h}=\sum_{A=1}^{N} u_{A} \mathcal{N}_{A}
$$


Using (5) in (4) and explicitly writing the resulting linear system

$$
\sum_{A=1}^{N}\left[\int_{\Omega}\left(\nabla \mathcal{N}_{A} \cdot \overline{\nabla \mathcal{N}_{B}}-k^{2} \mathcal{N}_{A} \overline{\mathcal{N}_{B}}\right) d \Omega+\mathrm{i} k \int_{\Gamma} \mathcal{N}_{A} \overline{\mathcal{N}_{B}} d \Gamma\right] u_{A}=\int_{\Gamma} \overline{\mathcal{N}_{B}} g d \Gamma
$$

for $B=1, \cdots, N$ and the coefficients $u_{A}$ need to be determined. Rewriting (6) in matrix notation

$$
\left(\boldsymbol{K}-k^{2} \boldsymbol{M}+i k \boldsymbol{C}\right) \boldsymbol{u}=\boldsymbol{A} \boldsymbol{u}=\boldsymbol{f}
$$

where, $\boldsymbol{K}$ is called the global stiffness matrix, $\boldsymbol{M}$ the global mass matrix, $\boldsymbol{C}$ the global damping matrix and $\boldsymbol{f}$ the global load vector. The global solution vector $\boldsymbol{u}$ is obtained by solving the linear system $\boldsymbol{A} \boldsymbol{u}=\boldsymbol{f}$ where $\boldsymbol{A}=\left(\boldsymbol{K}-k^{2} \boldsymbol{M}+i k \boldsymbol{C}\right)$ is the global coefficient matrix. In view of the decomposition of $\Omega$ as the sum of elements $E$, we can write the individual entries of the linear system as the sum of integrals over elements. Thus,

$$
\boldsymbol{A}_{A, B}=\sum_{E} \int_{E}\left(\nabla \mathcal{N}_{A} \cdot \overline{\nabla \mathcal{N}_{B}}-k^{2} \mathcal{N}_{A} \overline{\mathcal{N}_{B}}\right) d \Omega(E)+\mathrm{i} k \int_{\partial E} \mathcal{N}_{A} \overline{\mathcal{N}_{B}} d \Gamma(E)
$$

and

$$
f_{A}=\sum_{E} \int_{\partial E} \overline{\mathcal{N}_{B}} g d \Gamma(E)
$$

The classical FEM and IGA differ from this point onwards depending on the choice of basis functions $\mathcal{N}_{A}$ and the way the elements $E$ are constructed.

\subsection{Lagrange finite elements}

In this paper, for classical FEM, we consider rectangular Lagrange finite elements with degrees of freedom associated with their corresponding nodes. Let us denote the number of degrees of freedom for an element $E$ by ne. Consider $\mathcal{N}_{a}, a=1, \cdots$, ne as the basis functions associated with vertices $\boldsymbol{x}_{a}^{E}$ of element $E$. The basis functions $\mathcal{N}_{a}$ satisfy

$$
\mathcal{N}_{a}^{E}\left(\boldsymbol{x}_{b}^{E}\right)=\delta_{a b}
$$

where $\delta_{a b}$ is the Kronecker delta. We can create a correspondence between the index $a$ of local basis function $\mathcal{N}_{a}$ and the index $A$ for the global basis functions $\mathcal{N}_{A}$ via a 'connectivity' array [31]

$$
A=\operatorname{IEN}(a, e)
$$

Thus due to 10$)$ and in view of connectivity information for a given global index, computation of the entry, say $\boldsymbol{K}_{A, B}$, does not require us to generate the basis functions $\mathcal{N}_{A}$ and $\mathcal{N}_{B}$ globally. We can write a typical entry in global stiffness matrix as sum over individual elements

$$
\boldsymbol{K}_{A, B}=\sum_{e \in D} \int_{E_{e}} \nabla \mathcal{N}_{A} \cdot \overline{\nabla \mathcal{N}_{B}} d \Omega(E)=\sum_{e \in D} \int_{E_{e}} \nabla \mathcal{N}_{a}^{E_{e}} \cdot \overline{\nabla \mathcal{N}_{b}^{E_{e}}} d \Omega(E)
$$

with $D$ being a set of elements indices where $\mathcal{N}_{A}$ and $\mathcal{N}_{B}$ overlap. The entries for other matrices can be computed and assembled by following the same procedure. For the purpose of numerical 
integration, we define $\hat{E}$ as the reference element of which all the elements in $\mathcal{T}_{h}$ are images. The physical element $E$ and the reference element $\hat{E}$ are related by the affine map $F_{E}$ given by

$$
F_{E}: \hat{\boldsymbol{\xi}} \in \hat{E} \rightarrow F_{E}(\hat{\boldsymbol{\xi}})
$$

Assuming $F_{E}$ is invertible, we can map functions from $\hat{E}$ to $E$ and perform the integration. Consider an element level integral for stiffness matrix in 12 defined over physical element $E$ that we need to write for $\hat{E}$ :

$$
I=\int_{E} \nabla \mathcal{N}_{a}^{E_{e}} \cdot \overline{\nabla \mathcal{N}_{b}^{E_{e}}} d \Omega(E)=\int_{\hat{E}}\left(J^{-1} \nabla \hat{N}_{a}\right) \cdot\left(J^{-1} \overline{\nabla \hat{N}_{b}}\right) \operatorname{det}(J) d \Omega(\hat{E})
$$

where $J$ is the Jacobian of the transformation $F_{E}$. In the current study we set $\hat{E}$ to be a quadrilateral in the space $\hat{\boldsymbol{\xi}} \in[-1,1]^{2}$. The integrals on reference element $\hat{E}$ are performed by employing the standard Gauss-Legendre quadrature. In 1D, the Lagrange basis function of order $p$ is given by

$$
\mathcal{N}_{a}(\xi)=\prod_{\substack{a=1 \\ a \neq b}}^{n} \frac{\hat{\xi}-\hat{\xi}_{b}}{\hat{\xi}_{a}-\hat{\xi}_{b}}
$$

where $n=p+1$. The basis functions $\mathcal{N}_{a}$ are defined on the reference element in $\xi$ space. In $2 \mathrm{D}$, the $p^{\text {th }}$ order polynomial in $\hat{\boldsymbol{\xi}}$ space can be built by taking tensor product of two $p^{\text {th }}$ order polynomials in 1D. It is easy to verify from (15), that the Lagrange basis satisfies the partition of unity property and is interpolatory at node locations.

\subsection{NURBS finite elements}

IGA follows the same philosphy as FEM but they differ in terms of how the 'elements' or 'nodes' are defined and the type of basis functions used [44]. Since we use NURBS in both our IGA and PUIGA implementations, for the sake of completeness a brief discussion on the key aspects of IGA is in order. However, for a thorough discussion on theoretical and implementational aspects of IGA one can refer to 31,44 .

\subsection{1 knot vector}

A knot vector is the basic building block of IGA. It can be given in one-dimension as a sequence of non-decreasing set of coordinates

$$
\Xi=\left[\xi_{1}, \xi_{2}, \cdots, \xi_{n+p+1}\right]
$$

where, $\xi_{i}$ is the $i^{\text {th }}$ knot, $n$ the number of basis functions and $p$ the polynomial order. The knot vector $\Xi$ can be considered as defined over parametric space, $\tilde{\boldsymbol{\xi}}=(\xi)$ (or $\tilde{\boldsymbol{\xi}}=(\xi, \eta)$ in $2 \mathrm{D}$ ). The B-splines are then constructed over this parametric space formed by knots and they map this space into the physical space. The intervals created in the parametric space due to knot vector are what is known as knot spans. Adding a knot into already existing knot vector is called the knot insertion which is akin to the $h$-refinement in FEM. 


\subsubsection{NURBS basis}

The $p^{\text {th }}$ order B-spline basis in 1D is obtained from the Cox-deBoor recursion formula:

$$
\mathcal{N}_{i, p}(\xi)=\frac{\xi-\xi_{i}}{\xi_{i+p}} \mathcal{N}_{i, p-1}(\xi)+\frac{\xi_{i+p+1}-\xi}{\xi_{i+p+1}-\xi_{i+1}} \mathcal{N}_{i+1, p-1}(\xi)
$$

where,

$$
\mathcal{N}_{i, 0}(\xi)= \begin{cases}1, & \text { if } \xi_{i} \leq \xi \leq \xi_{i+1}, \\ 0, & \text { otherwise }\end{cases}
$$

The NURBS basis is the weighted version of B-spline basis. In order to create a NURBS basis in 2D, the 1D B-spline basis in (18) can be combined using tensor product to create a bi-variate NURBS basis.

$$
\mathcal{R}_{i, j}^{p, q}(\xi, \eta)=\frac{\mathcal{N}_{i, p}(\xi) \mathcal{M}_{j, q}(\eta) w_{i, j}}{\sum_{i}^{n} \sum_{j}^{m} \mathcal{N}_{i, p}(\xi) \mathcal{M}_{j, q}(\eta) w_{i, j}}
$$

where, $p$ and $q$ respectively are the polynomial orders for the 1D NURBS bases $\mathcal{N}_{i, p}(\xi)$ and $\mathcal{M}_{j, q}(\eta)$ with associated knot vectors $\Xi=\left\{\xi_{1}, \xi_{2}, \cdots, \xi_{n+p+1}\right\}$ and $\mathcal{H}=\left\{\eta_{1}, \eta_{2}, \cdots, \eta_{m+q+1}\right\}$. It is easy to see that for $p=0$ and $p=1$, the B-spline basis is the same as Lagrangian basis in (15). Consider that a knot value is repeated $m$ times in a given knot vector. In such case, NURBS basis is $C_{p-m}$ continuous. Whereas when the knot value appears just once in the knot vector the basis is $C_{p-1}$ continuous. The functions are $C^{\infty}$ within the knot span. Therefore using NURBS basis can give more accurate results compared to the Lagrangian basis.

\subsubsection{Geometry and functions representation with NURBS}

We express a surface in $2 \mathrm{D}$ as

$$
\mathbf{S}(\xi, \eta)=\sum_{i}^{m} \sum_{j}^{n} R_{i, j}^{p, q}(\xi, \eta) \mathbf{P}_{i, j}
$$

where $m$ and $n$, respectively, are the number of basis functions in $\xi$ and $\eta$ direction and $\mathbf{P}_{i, j}$ are the so called 'control points'. Throughout this paper, we use $m=n$. The user can choose the weights $w_{i, j}$ in (19) and the location of control points in order to build and manipulate the geometry. It can be noted that in order to define 'elements' in a NURBS based method, we consider the parametric area with non-zero values. Let $\mathcal{K}_{1}$ and $\mathcal{K}_{2}$ be the vectors with unique knot values respectively in $\xi$ and $\eta$ parametric coordinate, i.e.,

$$
\begin{aligned}
& \mathcal{K}_{1}=\left\{\xi_{i}: \xi_{i} \neq \xi_{i+1}, \text { for } i=1, \cdots, \operatorname{dim}\left(\mathcal{K}_{1}\right)\right\} \quad \text { and } \\
& \mathcal{K}_{2}=\left\{\eta_{i}: \eta_{i} \neq \eta_{i+1}, \text { for } i=1, \cdots, \operatorname{dim}\left(\mathcal{K}_{2}\right)\right\}
\end{aligned}
$$

An element $\tilde{\Omega}^{e}$ in parametric space can then be defined as

$$
\tilde{\Omega}^{e}=\left[\xi_{i}, \xi_{i+1}\right] \times\left[\eta_{j}, \eta_{j+1}\right]
$$

Here, $e$ is the index of the element and can be easily given as

$$
e=j\left(\operatorname{dim}\left(\mathcal{K}_{1}\right)-1\right)+i
$$


Recall that the NURBS basis functions are defined in parametric space and a transformation is necessary to map coordinates and functions to the 'physical space'. A third space, namely, the 'reference element space' is also essential in order to perform the numerical quadrature. We use the same reference element space as used in Lagrange FEM, i.e., the reference element for NURBS is again $\hat{E}=[-1,1]^{2}$ and denote corresponding coordinates in parent space as $\hat{\boldsymbol{\xi}}=(\hat{\xi}, \hat{\eta})$. We use same polynomial orders in $\xi$ and $\eta$ directions so we will now omit the superscripts $p$ and $q$ when writing the NURBS basis $R_{i, j}^{p, q}$. The approximation space for IGA is built with the same basis functions as those used for constructing the geometry. Assuming there are $N$ unknowns in the IGA system, we can approximate $u_{h}$ as

$$
u_{h}=\sum_{A=1}^{N} u_{A} R_{A}
$$

where the index $A=n(j-1)+i$. Substituting (25) in (4), we would obtain a similar linear system as in (6),

$$
\sum_{A=1}^{N}\left[\int_{\Omega}\left(\nabla R_{A} \cdot \overline{\nabla R_{B}}-k^{2} R_{A} \overline{\nabla R_{B}}\right) d \Omega+\mathrm{i} k \int_{\Gamma} R_{A} \overline{\nabla R_{B}} d \Gamma\right] u_{A}=\int_{\Gamma} \overline{R_{B}} g d \Gamma
$$

for $B=1, \cdots, N$ and $u_{A}, A=1, \cdots, N$ are the degrees of freedom that need to be determined. It is clear that the matrix representation of (26) would be same as (7) except the underlying basis functions are NURBS. Writing (26) in matrix notation

$$
\left(\boldsymbol{K}^{*}-k^{2} \boldsymbol{M}^{*}+\mathrm{i} k \boldsymbol{C}^{*}\right) \boldsymbol{u}^{*}=\boldsymbol{A}^{*} \boldsymbol{u}^{*}=\boldsymbol{f}^{*}
$$

The integrals expressed in (26) are over the physical domain $\Omega$ and their evaluation is performed 'element' wise with the corresponding entries added to the global matrix. The 'element' in IGA is the tensor product parametric space in $(23)$, formed due to non-zero knot intervals. Let us assume for now that the NURBS basis can be written in parent coordinates $\hat{\boldsymbol{\xi}}$ and that we can establish the mappings: $F_{1}: \hat{\Omega}^{e} \rightarrow \tilde{\Omega}^{e}$ and $F_{2}: \tilde{\Omega}^{e} \rightarrow \Omega^{e}$. The function $u_{h}$ can be approximated as in parent coordinates as

$$
u_{h}^{e}(\hat{\boldsymbol{\xi}})=\sum_{a=1}^{n e} u_{a}^{e} R_{a}^{e}(\hat{\boldsymbol{\xi}})
$$

where $u_{a}^{e}$ and $R_{a}^{e}$ with $a=1, \cdots$, ne are respectively the control variables and basis functions that are local to the element $e$. Note that $n e=(1+p)^{2}$ is the number of non-zero basis functions in $e$. A typical entry in the local stiffness matrix therefore can be written as

$$
k_{a, b}^{e}=\int_{\hat{E}} \nabla R_{a}^{e} \cdot \overline{\nabla R_{b}^{e}} \operatorname{det}(J) d \hat{E}
$$

Here, $\operatorname{det}(J)$ is the determinant of Jacobian which involves two mappings, i.e., $J=\left|F_{1}\right|\left|F_{2}\right|$. A similar mapping as in (11) between indices of the global and local basis functions can be generated for the NURBS basis. Using the connectivity information from IEN array, we can assemble the local entry in 29) into the global stiffness matrix $\boldsymbol{K}^{*}$ in (27). Solving (27) and then recombining the solution vector $\boldsymbol{u}^{*}$ with basis function using (25) gives the IGA approximation for $u_{h}$. 


\subsection{Plane wave enrichment}

In both PUFEM [11] and PUIGA [29], $u_{h}$ is approximated with the respective polynomial basis functions multiplied with a set of plane waves. The amplitudes of these plane waves are the unknowns and replace the unknown nodal (in Lagrangian FEM) or control point values (in IGA). We define the set of plane waves as

$$
\psi_{Q}=\operatorname{span}\left\{e^{\mathrm{i} k\left(x \cos \left(\theta_{q}\right)+y \sin \left(\theta_{q}\right)\right)}, q=1,2, \ldots, Q\right\}
$$

The plane wave directions are evenly spaced around the unit circle. Let $\varphi_{i}, i=1, \cdots, N$ denote the polynomial basis functions. The partition of unity (PU) space is then defined as

$$
V_{h}=\operatorname{span}\left\{\varphi_{A} e^{\mathrm{i} k\left(x \cos \left(\theta_{q}\right)+y \sin \left(\theta_{q}\right)\right)}, A=1,2, \ldots, N, q=1,2, \ldots, Q\right\}
$$

We can approximate $u_{h}$ with the above PU space as

$$
u_{h}^{Q}=\sum_{A=1}^{N} \varphi_{A} \sum_{q=1}^{Q} s_{A, q} \psi_{A, q}
$$

where $s_{A, q}$ is the amplitude of $q^{\text {th }}$ plane wave $\psi_{A, q}$ associated with the polynomial basis function $\varphi_{A}$. We denote the PU approximation of $u_{h}$ with superscript $Q$ only to distinguish it from purely polynomial based approximations in (5) or (25). Let us define a 'modified' shape function, say $\chi_{\alpha}$, which is a product of the $q^{\text {th }}$ plane wave with the basis function with global index $A$, i.e., $\chi_{\alpha}=\varphi_{A} e^{\mathrm{i} k\left(x \cos \left(\theta_{q}\right)+y \sin \left(\theta_{q}\right)\right)}$. If there are a total of $N$ basis functions each associated with $Q$ plane waves (see (32)) then the approximation for $u_{h}^{Q}$ in 32 can be written as

$$
u_{h}^{Q}=\sum_{\alpha=1}^{N Q} \chi_{\alpha} s_{\alpha}
$$

where $n$ tot $=N Q$ is size of the PU space and $\alpha=(A-1) Q+q$. Finally, using (33) in (4) to write the linear system for PU based methods

$$
\sum_{\alpha=1}^{n t o t}\left[\int_{\Omega}\left(\nabla \chi_{\alpha} \cdot \overline{\nabla \chi_{\beta}}-k^{2} \chi_{\alpha} \overline{\nabla \chi_{\beta}}\right) d \Omega+\mathrm{i} k \int_{\Gamma} \chi_{\alpha} \overline{\nabla \chi_{\beta}} d \Gamma\right] s_{\alpha}=\int_{\Gamma} \overline{\chi_{\beta}} g d \Gamma
$$

for a set of $n$ tot equations and a total of ntot unknowns $s_{\alpha}, \alpha=1, \cdots$, ntot and where $\beta=1, \cdots$, ntot. Note that the unknowns $s_{\alpha}$ are in fact are the plane wave amplitudes. Setting the basis functions $\varphi_{A}$ in (32) respectively as Lagrangian or NURBS polynomials, we obtain either a PUFEM or PUIGA implementation. All the integrals in (34) are evaluated with high order Gauss quadrature where around ten integration points are used per-direction per-wavelength. This is important in order to evaluate the oscillatory integrand over the multi-wavelength-sized elements. It should be noted that despite the relatively high number of integration points both PUFEM and PUIGA are still a lot more efficient to solve compared to the standard FEM. This is mainly due to the significant reduction in the total number of elements and the corresponding reduction in the total number of degrees of freedom. 


\section{$4 \quad$ Numerical results}

\subsection{Acoustic plane wave scattering}

In the first test case we consider the scattering of a unit amplitude plane wave by a rigid circular scatterer. For a plane wave travelling in the positive $x$-direction, the analytical solution for the total acoustic potential is given by:

$$
u=-\sum_{l=0}^{L} \mathrm{i}^{1} \epsilon_{l} \frac{J_{l}^{\prime}(k a)}{H_{l}^{\prime}(k a)} H_{l}(k r) \cos (l \theta)
$$

where $\epsilon_{l}=1$ if $l=0$ otherwise $\epsilon_{l}=2$, while $J_{l}(k a)$ and $H_{l}(k a)$ are, respectively, the Bessel and the Hankel functions of the first kind and order $l$. The prime in $J_{l}^{\prime}(k a)$ denotes the derivative of the Bessel function with respect to its argument. The remaining parameters are: the radius of the scattering circle $a$ and the polar coordinates $\theta$ and $r$. To avoid numerical errors coming from approximate boundary conditions we impose the analytical solution of the scattering problem (35) on the domain boundary using the Robin boundary condition $(1 \mathrm{~b})$. Here, we use the weak formulation in (2) with the function $g=\frac{\partial u}{\partial \mathbf{n}}+\mathrm{i} k u$ evaluated using the analytical solution in (35). The circular scatterer is centred at the origin $[0,0]$ while the computational domain is taken as a unit square centred at $[5,5]$. We measure the accuracy of the numerical solution $u_{h}$ by computing the relative errors in $H^{1}$-norm. Given that the exact solution $u$ for a problem under consideration is known, the relative error is evaluated by

$$
H^{1} \text {-error }=\frac{\left\|u-u_{h}\right\|_{H^{1}(\Omega)}}{\|u\|_{H^{1}(\Omega)}}
$$

where the $H^{1}$-norm for the Helmholtz equation is

$$
\|v\|_{H^{1}(\Omega)}=\|\nabla v\|_{L^{2}(\Omega)}^{2}+k^{2}\|v\|_{L^{2}(\Omega)}^{2}
$$

In order to evaluate the pollution we also need to calculate the $H^{1}$ projection or the best approximation of the exact solution $u$ in the approximation space $V_{h}$

$$
u_{h}^{b a}=\underset{u_{h} \in V_{h}}{\arg \min }\left\|u-u_{h}\right\|
$$

The best approximation $u_{h}^{b a}$ can then be computed by solving the following variational problem: find $u_{h}^{b a} \in V_{h}$, s.t.,

$$
\int_{\Omega}\left(\nabla u_{h}^{b a} \overline{\nabla v_{h}}+k^{2} u_{h}^{b a} \overline{v_{h}}\right) d \Omega=\int_{\Omega}\left(\nabla u \overline{\nabla v_{h}}+k^{2} u \overline{v_{h}}\right) d \Omega
$$

A similar discretization procedure as in Section 3 can be followed to form the linear system corresponding to the weak form in (39). Choosing the appropriate basis, either polynomial or enriched, gives us the corresponding best approximation in a given discrete space. To compare the various methods considered for their ability to handle pollution we compare the errors for the best approximation and to their respective Galerkin errors for a purely polynomial or PU based space. 

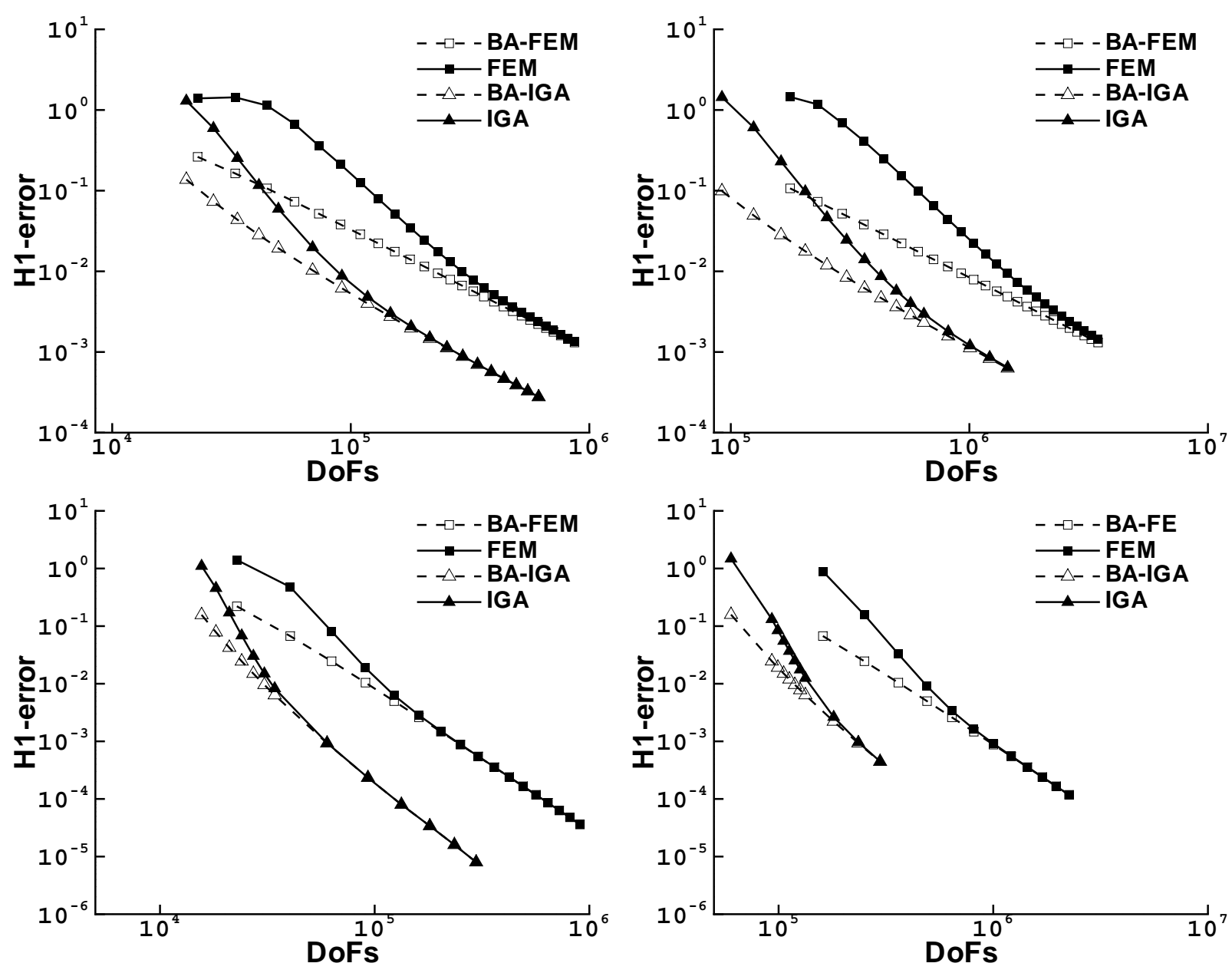

Figure 1: IGA vs FEM H1-norm error plotted against total number of degrees of freedom for $p=3$ (top row) and $p=5$ (bottom row) for the considered wavenumbers $k=100 \pi$ (left column) and $k=200 \pi$ (right column).

\subsubsection{Pollution error with non-enriched basis}

Our first aim in this example is to study the pollution error with non-enriched basis functions. The problem is solved using Lagrange as well as NURBS finite elements. Figure 1 shows a comparison of the errors obtained with each method for $k=100 \pi$ and $200 \pi$. These errors are plotted against the number of degrees of freedom (DoF) for $h$-refined mesh grids. Basis functions of order three i.e. $p=3$ are used to approximate the solution with both methods. The best approximation results of each method are also plotted in the same figure with dashed lines.

The significant difference between the solution errors and the best approximation clearly indicates the pollution effect for both considered methods. As finer meshes are considered the pollution effect becomes less pronounced until it eventually disappears. This can be seen for both type of elements where the plots show the solution errors converge toward the best approximation for finer meshes. However, the NURBS based elements show better performance in this regard where the solution error converges toward the best approximation at a much lower number of degrees of freedom compared to FEM. For example for $k=100 \pi$ the IGA error becomes similar to the best approximation error at around DoFs $=3 \times 10^{5}$ while with polynomial based finite elements the two errors meet at around $\mathrm{DoFs}_{\mathrm{s}}=8 \times 10^{5}$. For the higher considered wavenumber i.e. $k=200 \pi$ the pollution affects the results to a much higher DoFs compared to $k=100 \pi$. This is expected as the pollution error increases with 
higher wavenumbers. Here the IGA solution error converges to the best approximation at around DoFs $=14.5 \times 10^{5}$ while for FEM at around DoFs $=40 \times 10^{5}$. To understand the effect of increasing the polynomial order on this behaviour the same results are also shown in Figure 1 but for $p=5$. It can be seen that the pollution impact is reduced with the higher $p$. For example for $k=100 \pi$ the errors obtained with IGA and the best approximation meet at around DoFs $=0.6 \times 10^{5}$ while for FEM this number increases to DoFs $=2.5 \times 10^{5}$. Again increasing the wavenumber to $k=200 \pi$ causes a delay in the errors converging toward the best approximation until around DoFs $=3 \times 10^{5}$ for IGA and DoFs $=12.1 \times 10^{5}$ for FEM. As it can be seen in Figure 1, the best approximation and Galerkin errors for both IGA as well as FEM approach the theoretical rates of convergence when the meshes are sufficiently refined to the point where the pollution is vanished. However, for the same number of degrees of freedom the results with IGA shows an order of magnitude improvement in the error compared to FEM.

\subsubsection{Pollution error with enriched basis}

Next, we want to examine the performance of the enriched basis functions. The NURBS and the polynomial basis functions are enriched with plane waves. The aim is to check if PUIGA retains the same advantages observed with IGA in terms of degrees of freedom and the pollution error. We again solve the scattering problem for two different wavenumbers, namely, $k=100 \pi$ and $k=200 \pi$. The considered weighting functions are NURBS or Lagrange polynomials of order three $p=3$ enriched with 20 or 40 plane waves $(q=20$ or 40$)$. The problem is solved on a sequence of refined mesh grids. Figure 2 shows the solution as well as the best approximation errors for both PUIGA and PUFEM. Clearly, increasing the wavenumber from $100 \pi$ to $200 \pi$ requires increasing the number of degrees of freedom to achieve the same accuracy. This is observed with $q=20$ and 40 for PUIGA as well as PUFEM. Also increasing the number of enriching plane waves from $q=20$ to 40 reduces the number of degrees of freedom required to achieve a given accuracy for both enriched methods. With regard to the pollution error and for $q=20$ both methods seems to suffer from the pollution effect at much lower errors compared to the non-enriched method. For example in the previous set of results for $k=100 \pi$ the pollution affects the IGA and the FEM results up to an error of around $10^{-3}$ while again for $k=100 \pi$ and $q=20$ the pollution seems to affect PUIGA and PUFEM results even after an error of around $10^{-5}$. Obviously the same errors are achieved at a much smaller number of degrees of freedom with enriched approaches than with non-enriched. Nevertheless, the pollution impact can still be seen at a much higher accuracy with enriched methods. Furthermore, when increasing the number of enrichment functions to $q=40$ with both PUFEM and PUIGA the error does not converge toward the best approximation error at all. This can be an indication that at higher numbers of enrichment functions the solution will always suffer from the pollution. However, it also must be noted that in all the cases the error with PUIGA seems to be in general smaller than that with PUFEM and the difference between PUIGA error and the best approximation error is smaller than that of PUFEM and its best approximation. This suggests that PUFEM is more vulnerable to the pollution than PUIGA.

To investigate the results further we also plot in Figure 3 the condition numbers that correspond to the errors plots. As often reported on enriched methods it can be seen that high condition numbers are associated with PUIGA and PUFEM. The condition numbers grow rapidly as the number of degrees of freedom is increased. For a given number of degrees of freedom increasing the wavenumber form $k=100 \pi$ to $200 \pi$ reduces the condition number. Also increasing the number of plane waves from $q=20$ to 40 leads to a steeper increase in the condition numbers. In general the condition numbers of PUIGA results and its best approximation are similar and the same can be observed for 

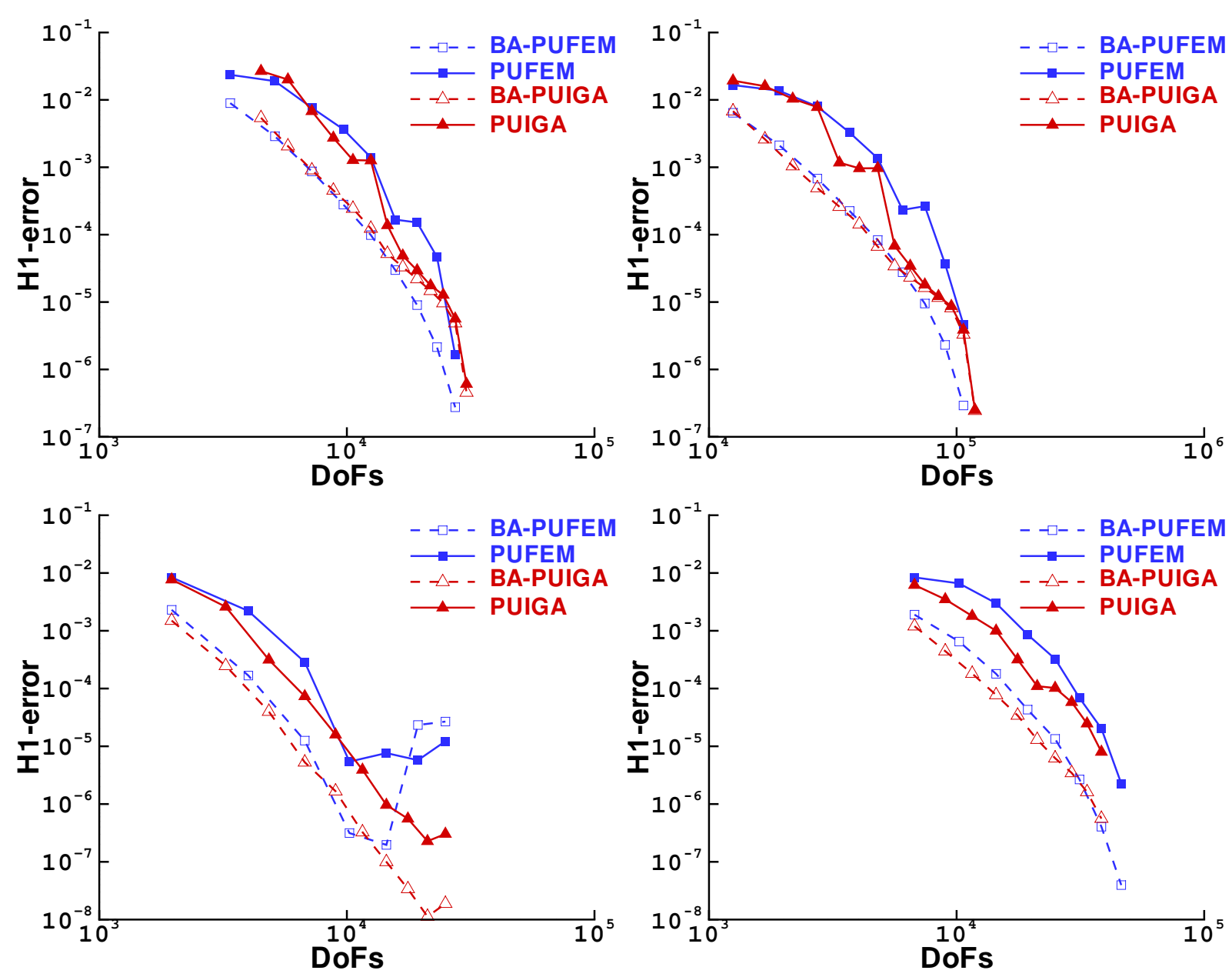

Figure 2: PUIGA vs PUFEM H1-norm error for $k=100 \pi$ (left column) and $k=200 \pi$ (right column) with $p=3$ and $q=20$ (first row) and $q=40$ (second row).

PUFEM. At lower number of degrees of freedom PUIGA leads to higher condition numbers than PUFEM. This is reversed at higher numbers of degrees of freedom where PUFEM is associated with higher condition numbers.

Our last concern in this subsection is to investigate the effect of increasing the polynomial order on enriched methods. To this end we consider again the scattering problem for $k=100 \pi$ and $k=200 \pi$. We solve the problem using PUIGA and PUFEM with $q=20$. However, we now increase the polynomial order to $p=5$. Figure 4 shows the errors plotted against an increased total number of degrees of freedom. The pollution effect on the results is still evident in the new set of results. Again the results obtained with PUIGA seems to suffer less pollution compared to PUFEM results specifically for the higher wavenumber i.e. $k=200 \pi$. The convergence of PUIGA error towards its best approximation error is happening now at an error of around $10^{-6}$ compared to $10^{-5}$ for $p=3$. The results shows that the number of degrees of freedom required to achieve a certain accuracy is in general lower for $p=3$ and $q=40$. For example in the case $k=100 \pi$ PUIGA $(p=3$ and $q=40)$ the solution with 11560 degrees of freedom has an error of $3.91 \times 10^{-6}$ while for PUIGA $(p=5$ and $q=20$ ) the solution with 12500 degrees of freedom has an error of $2.72 \times 10^{-4}$. This is around two orders of magnitude difference. However, the average convergence rate for PUIGA ( $p=3$ and $q=40)$ when $k=100 \pi$, is around 5.0 compared to around 6.8 for PUIGA $(p=5$ and $q=20)$. These results suggest that using a higher $p$ with a smaller $m$ can lead to a higher convergence rate. 

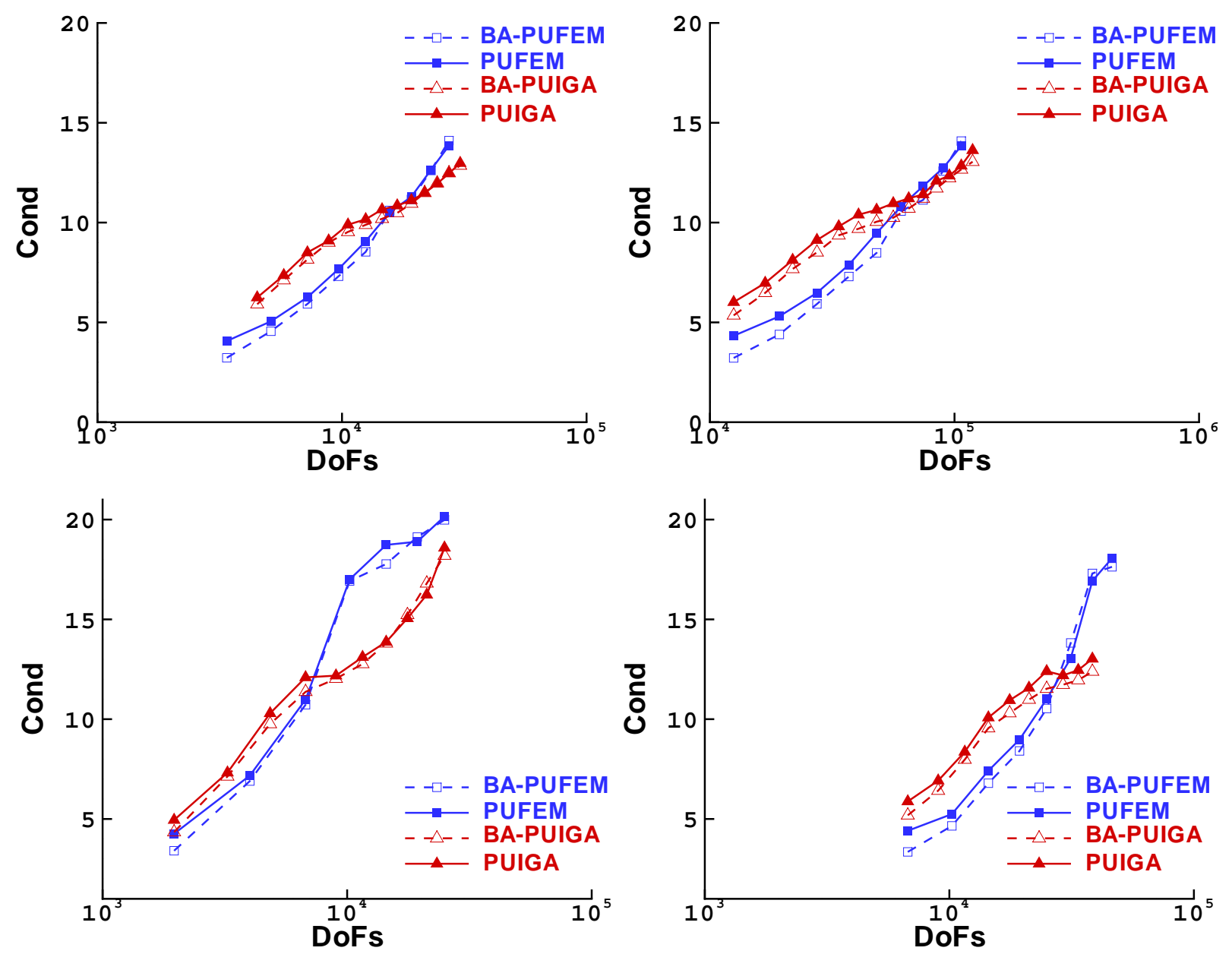

Figure 3: PUIGA vs PUFEM log of the condition numbers for $k=100 \pi$ (left column) and $k=200 \pi$ (right column) with $p=3$ and $q=20$ (first row) and $q=40$ (second row).

Observations similar to PUIGA may also be made for PUFEM. For $k=200 \pi$ and PUFEM $(p=3$ and $q=40$ ) the solution with 19360 degrees of freedom has an error of $3.78 \times 10^{-4}$ while for PUFEM $(p=5$ and $q=20)$ the solution with 19220 degrees of freedom has an error of $1.54 \times 10^{-2}$. Again similar to PUIGA the convergence rate of PUFEM $(p=5$ and $q=20)$ is higher than PUFEM ( $p=3$ and $q=40$ ) where the later rate is around 4 while the former is above 7 . But it should also be noted that PUFEM seems to be badly affected with the poor conditioning issue for $p=5$ and $q=20$. This can be seen in Figure 4 where PUFEM starts to diverge at the end of the error curves unlike PUIGA which seems to be more stable for the considered range of degrees of freedom.

\subsubsection{Empirical quasi-optimality estimates}

Although we have shown that in general IGA and PUIGA, respectively, outperforms Lagrangian FEM and PUFEM, they are not completely free from pollution. It is well known that the 'rule of thumb' of 8-10 dofs per wavelength can ensure bounded errors for the best approximation. However, the constant resolution following the rule of thumb cannot guarantee a bounded error for FEM based computations. In order to achieve quasi-optimal errors (i.e. no dispersion) from FEM, $k(k h)^{p}$ has to be kept constant, see [6]. Although this gives decreasing errors, such a condition will require highly refined meshes as $k$ grows. In engineering practice, it is generally sufficient if the errors can be 

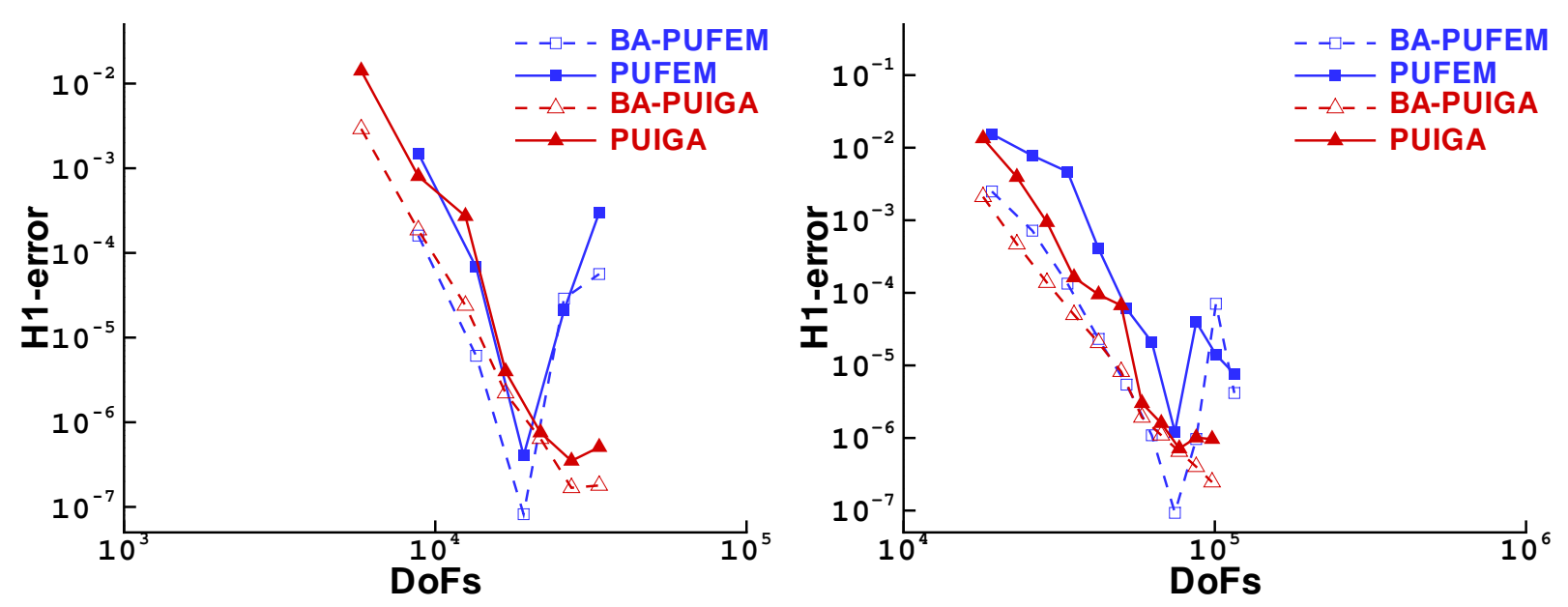

Figure 4: PUIGA vs PUFEM H1-norm error plotted against the total number of degrees of freedom for $p=5$ and $q=20$ for $k=100 \pi$ (left column) and $200 \pi$ (right column).

bounded, say between 0.1 to $1 \%$. Thus we are more interested in the case where the pollution may well be present and the gap (or rather the ratio) between the Galerkin error and best approximation error increases with the wavenumber as predicted by theory [2]. To this end the quasi-optimality constant is considered the measure of pollution and is given by the ratio of Galerkin and best approximation errors, i.e.,

$$
C_{q}=\frac{\left\|u-u_{h}\right\|_{H^{1}(\Omega)}}{\left\|u-u_{b a}\right\|_{H^{1}(\Omega)}}
$$

It is shown in Ihlenburg [2] that the constant $C_{q}$ grows with $k$ in the preasymptotic range when using constant resolution. The wavenumber dependence of the 'quasi-optimality' constant in the preasymptotic range can therefore quantify how sensitive a given method is to the pollution effect. In this section, we will study all four considered methods for the scattering problem defined in Test case 1 with $p=3$ and $p=5$. We compare FEM and IGA for wavenumbers $25 \pi, 50 \pi, 100 \pi$ and $200 \pi$ whereas PUFEM and PUIGA for $50 \pi, 100 \pi, 200 \pi$ and $300 \pi$. Table 1 shows the $H^{1}$-errors from FEM and IGA as well as the errors from the corresponding best approximations. At this point, it is convenient to define the number of degrees of freedom per wavelength through a parameter:

$$
\tau=\lambda \sqrt{n D o F}
$$

where $\lambda=2 \pi / k$ is the wavelength and $n D o F$ the total number of degrees of freedom in the problem. Note that for FEM and IGA, $n D o F=N$ whereas for PUFEM and PUIGA $n D o F=N Q$. For FEM and IGA we constrain $\tau \approx 8$ when $p=3$ and $\tau \approx 6.5$ when $p=5$. These values of $\tau$ are chosen in order to ensure the $H^{1}$-errors from FEM remain between $1-5 \%$. We keep the same $\tau$ for IGA computations. As seen from Table 1, IGA improves the accuracy of numerical solution compared to FEM for the same value of $\tau$. The gain in accuracy with IGA for $p=3$ is roughly one order of magnitude whereas that for $p=5$ is around two orders of magnitude. The quasi-optimality constant $C_{q}$ grows with wavenumber for $p=3$ for both IGA and FEM as predicted by theory. We expect the higher order method, for both FEM and IGA, to suffer less from the pollution. The growth rates for FEM and IGA with $p=3$ are $C_{q} \propto k^{0.57}$ and $C_{q} \propto k^{0.11}$, respectively. When the polynomial order is increased to $p=5$, we get $C_{q} \propto k^{0.49}$ and $C_{q} \propto k^{0.004}$ for FEM and IGA, respectively. Thus it is evident that for both FEM and IGA, the value of $C_{q}$ drops for a given wavenumber when moving from $p=3$ to $p=5$. Also, for IGA with $p=5, C_{q}$ increases very slowly 
which indicate that the smoothness of NURBS basis functions due to higher order plays a significant role in dealing with the pollution. For PUFEM and PUIGA, we constrain $\tau \approx 1$ and study $p=3$

\begin{tabular}{|c|c|c|c|}
\hline$k$ & & FEM & IGA \\
\hline \multirow{3}{*}{$25 \pi$} & a & $4.9427 \mathrm{E}-02$ & $2.8490 \mathrm{E}-03$ \\
& b & $3.1585 \mathrm{E}-02$ & $2.8292 \mathrm{E}-03$ \\
& c & 1.5649 & 1.0070 \\
\hline \multirow{3}{*}{$50 \pi$} & a & $8.2904 \mathrm{E}-02$ & $2.4792 \mathrm{E}-03$ \\
& b & $3.1600 \mathrm{E}-02$ & $2.4281 \mathrm{E}-03$ \\
& c & 2.6236 & 1.0211 \\
\hline \multirow{3}{*}{$100 \pi$} & a & $5.1634 \mathrm{E}-02$ & $3.0278 \mathrm{E}-03$ \\
& b & $1.7533 \mathrm{E}-02$ & $2.7418 \mathrm{E}-03$ \\
& $\mathrm{c}$ & 2.9450 & 1.1043 \\
\hline \multirow{3}{*}{$200 \pi$} & a & $9.8605 \mathrm{E}-02$ & $2.9614 \mathrm{E}-03$ \\
& b & $1.7533 \mathrm{E}-02$ & $2.3136 \mathrm{E}-03$ \\
& $\mathrm{c}$ & 5.6241 & 1.2800 \\
\hline
\end{tabular}

\begin{tabular}{|c|c|c|c|}
\hline$k$ & & FEM & IGA \\
\hline \multirow{3}{*}{$25 \pi$} & a & $2.0050 \mathrm{E}-02$ & $2.1480 \mathrm{E}-04$ \\
& b & $1.7018 \mathrm{E}-02$ & $2.1479 \mathrm{E}-04$ \\
& c & 1.1782 & 1.0001 \\
\hline \multirow{3}{*}{$50 \pi$} & a & $2.6915 \mathrm{E}-02$ & $2.3362 \mathrm{E}-04$ \\
& b & $1.7027 \mathrm{E}-02$ & $2.3358 \mathrm{E}-04$ \\
& $\mathrm{c}$ & 1.5808 & 1.0002 \\
\hline \multirow{3}{*}{$100 \pi$} & a & $4.5094 \mathrm{E}-02$ & $2.3431 \mathrm{E}-04$ \\
& b & $1.7031 \mathrm{E}-02$ & $2.3414 \mathrm{E}-04$ \\
& $\mathrm{c}$ & 2.6478 & 1.0007 \\
\hline \multirow{3}{*}{$200 \pi$} & $\mathrm{a}$ & $3.2793 \mathrm{E}-02$ & $4.4876 \mathrm{E}-04$ \\
& b & $1.0433 \mathrm{E}-02$ & $4.4419 \mathrm{E}-04$ \\
& $\mathrm{c}$ & 3.1433 & 1.0103 \\
\hline
\end{tabular}

Table 1: FEM vs IGA: empirical $C_{q}$ for $p=3$ (left) and $p=5$ (right); a: $H^{1}$-error, b: $H^{1}$-error in BA, c: $C_{q}$.

with $Q=8$. As seen from Table 2, PUFEM and PUIGA exhibit $C_{q} \propto k^{0.58}$ and $C_{q} \propto k^{0.028}$ respectively with $\tau \approx 1$. As expected, for both PUFEM and PUIGA, we significantly gain in terms of accuracy for a $\tau$ much smaller than for the corresponding un-enriched method. For PUFEM with $p=3, C_{q}$ still grows with $k$ whereas for PUIGA it is roughly constant with some fluctuation. Now we consider the case where the mesh is refined such that the mesh width $h$ satisfies

\begin{tabular}{|c|c|c|c|c|}
\hline$k$ & $\tau$ & & PUFEM & PUIGA \\
\hline \multirow[t]{3}{*}{$50 \pi$} & \multirow[t]{3}{*}{1.13} & $\mathrm{a}$ & $1.40 \mathrm{E}-03$ & $1.51 \mathrm{E}-04$ \\
\hline & & $\mathrm{b}$ & $1.84 \mathrm{E}-04$ & $3.62 \mathrm{E}-05$ \\
\hline & & $\mathrm{c}$ & 7.61 & 4.16 \\
\hline \multirow[t]{3}{*}{$100 \pi$} & \multirow[t]{3}{*}{1.07} & $\mathrm{a}$ & $1.30 \mathrm{E}-03$ & $5.21 \mathrm{E}-05$ \\
\hline & & b & $1.15 \mathrm{E}-04$ & $1.26 \mathrm{E}-05$ \\
\hline & & $\mathrm{c}$ & 11.27 & 4.14 \\
\hline \multirow[t]{3}{*}{$200 \pi$} & \multirow[t]{3}{*}{1.05} & $\mathrm{a}$ & $1.40 \mathrm{E}-03$ & $2.76 \mathrm{E}-05$ \\
\hline & & $\mathrm{b}$ & $8.24 \mathrm{E}-05$ & $6.74 \mathrm{E}-06$ \\
\hline & & $\mathrm{c}$ & 16.99 & 4.09 \\
\hline \multirow{3}{*}{$300 \pi$} & \multirow[t]{3}{*}{1.04} & $\mathrm{a}$ & $1.50 \mathrm{E}-03$ & 2.11E-05 \\
\hline & & $\mathrm{b}$ & $6.88 \mathrm{E}-05$ & $5.11 \mathrm{E}-06$ \\
\hline & & $\mathrm{c}$ & 21.81 & 4.13 \\
\hline
\end{tabular}

Table 2: PUFEM vs PUIGA: empirical $C_{q}$ for $p=3$; a: $H^{1}$-error, b: $H^{1}$-error in BA, c: $C_{q}$.

$h k^{\alpha}=C$ and the $H^{1}$ errors obtained from the finite elements and isogeometric analysis are bounded. For this we consider the same wavenumbers as in Table 1 and 2 for the methods considered. With the polynomial order $p=3$ and using $\tau \approx 6$, the average $H^{1}$ errors are $\mathbf{8 . 3 \%}$ and $\mathbf{0 . 2 \%}$ for FEM and IGA, respectively. For $p=5$ the average $H^{1}$ errors are reduced to $5 \%$ for FEM and $0.02 \%$ for IGA. For enriched approaches, we 

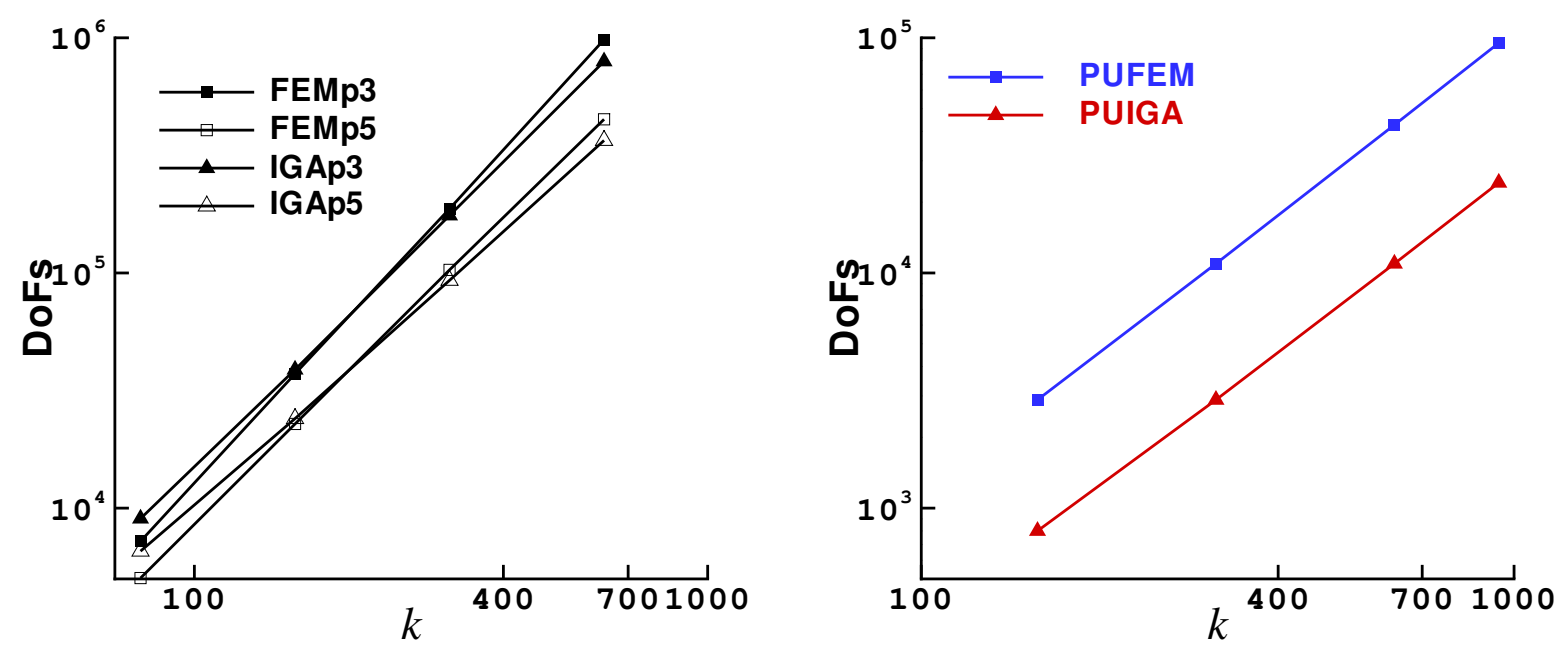

Figure 5: Degree of freedom growth for bounded errors: total number of degrees of freedom against increasing wavenumber for FEM and IGA polynomial orders $p=3$ and 5 (left) and PUFEM and PUIGA with $p=3$ (right).

consider the mesh refinement with $p=3$ and $Q=8$. The average $H^{1}$ error is then of the order $\mathbf{0 . 0 0 1 \%}$ with both PUFEM and PUIGA. But it should be noted that significantly fewer degrees of freedom are needed for PUIGA than for PUFEM to achieve this level of errors. The numerical experiments indicate that for $p=3$, we require $\alpha=1.2$ for FEM and $\alpha=1.1$ for IGA. The requirement on $\alpha$ is less demanding as polynomial order $p$ is increased to 5 . In the case with $p=5$, FEM gives bounded errors for $\alpha=1.1$ and IGA for $\alpha=1.0$. These numerical experiments are best described by the plot showing the growth in number degrees of freedom $(n D o F)$ as a function of wavenumber $(k)$ in Figure 5. For FEM with $p=3$ and $p=5$, the required growth rates to get bounded $H^{1}$ errors are $n D o F \propto k^{2.36}$ and $k^{2.16}$, respectively. Whereas, IGA exhibits lower growth rates than FEM for both considered polynomial orders where the rates are $n D o F \propto k^{2.15}$ for $p=3$ and $n D o F \propto k^{1.98}$ for $p=5$. The PUIGA also results in a slightly slower growth rate than PUFEM where the rate is $n D o F \propto k^{1.90}$ with PUIGA while it is $n D o F \propto k^{1.95}$ with PUFEM. Although both PUFEM and PUIGA exhibit similar growth rates, PUIGA requires significantly less degrees of freedom to achieve bounded $H^{1}$ errors of the order $\mathbf{0 . 0 0 1 \%}$. These observations are in-line with the trends observed in Figure 2 for $p=3$.

\subsection{Car interior acoustics}

In the last test case we aim to evaluate the performance of PUIGA in recovering the interior noise in a 2D car cabin model shown in Figure 6. The car interior is reproduced from [45] where the details of the NURBS geometry description can be found. Unlike the first example where the domain is created using a single patch, in this example multiple patches are necessary to discretize the car interior geometry. Such complex geometries are common in many engineering applications. Therefore, it is important to test PUIGA convergence with multiple patch applications. In this example we build each patch with biquadratic B-splines. Thus, the interpolation within each patch is $C^{1}$ continuous. In order to ensure the inter-patch $C^{0}$ continuity, identical level of knot refinement has to be maintained across the patch interfaces. 

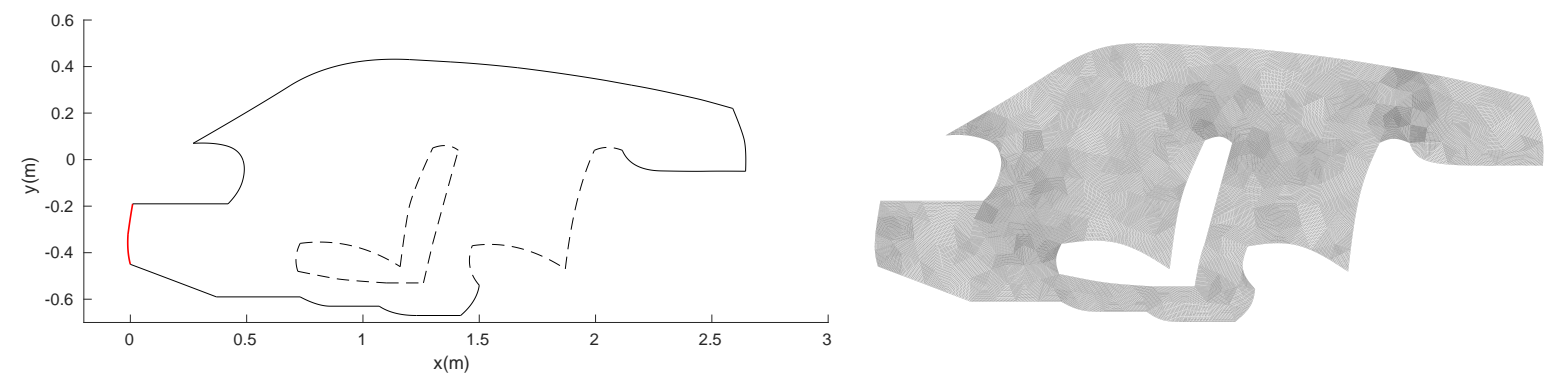

Figure 6: Car interior acoustics: geometry (left) and the considered mesh for FEM reference solution (333568 nodes and 165888 elements)
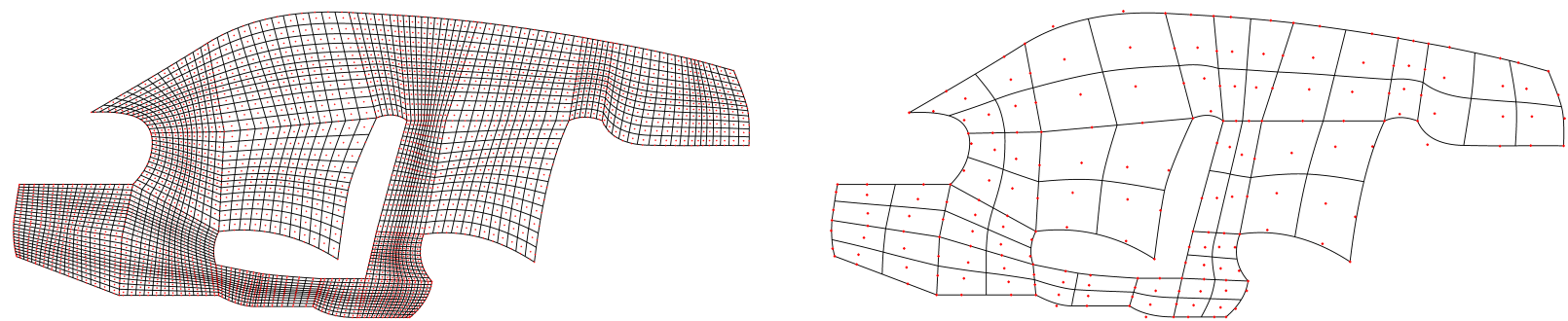

Figure 7: Car interior acoustics: IGA $(\mathrm{nCpt}=3521$ and patches $=19)$ and PUIGA $(\mathrm{nCpt}=211$, patches $=19$ and $q=8$ ) considered mesh grids.

The acoustic waves inside the cabin are assumed to be generated by the engine vibrations and transmitted through a part of the boundary $\left(\Gamma_{N}\right)$ depicted in red in Figure 6 . For simplicity we assume that the domain exterior boundaries are infinitely rigid compared to the air inside the cabin. Therefore the acoustic waves are fully reflected on these boundaries. However, the car seats depicted with dotted lines in Figure 6] are assumed to be made of an acoustically soft material. Thus, impedance boundary condition is imposed on this part of the domain boundary $\left(\Gamma_{Z}\right)$. For the domain boundary that is not part of both $\Gamma_{N}$ and $\Gamma_{Z}$, we assume a sound hard condition, i.e., $\frac{\partial u}{\partial n}=0$. The boundary conditions on $\Gamma_{N}$ (Neumann), and $\Gamma_{Z}$ (impedance i.e. the first-order absorbing boundary condition) are given by

$$
\begin{aligned}
& \frac{\partial u}{\partial n}=-2 \pi \mathrm{i} f \rho_{\text {air }} v_{n} \text { on } \Gamma_{N} \\
& \frac{\partial u}{\partial n}=-2 \pi \mathrm{i} f \rho_{\text {air }} \frac{u}{Z_{0}} \text { on } \Gamma_{Z} .
\end{aligned}
$$

where $f$ is the frequency of the acoustic wave, $\rho_{\text {air }}$ the density of air, $v_{n}$ the imposed normal velocity and $Z_{0}$ the imposed normal acoustic impedance. The considered values are $\rho_{\text {air }}=1.225 \mathrm{~kg} \mathrm{~m}^{-3}, v_{n}$ $=1 \mathrm{~m} \mathrm{~s}^{-1}$ and $Z_{0}=2000$ ryal. Since the domain boundary $\Gamma$ is split into $\Gamma_{N}$ and $\Gamma_{Z}$, the weak form for this specific problem is,

$$
\int_{\Omega}\left(\nabla u \overline{\nabla v}-k^{2} u \bar{v}\right) d \Omega+\int_{\Gamma_{Z}} Z u \bar{v}=\int_{\Gamma_{N}} g_{N} \bar{v}
$$

where $g_{N}=-2 \pi \mathrm{i} f \rho_{a i r} v_{n}$ and $Z=2 \pi \mathrm{i} f \rho_{\text {air }} \frac{1}{Z_{0}}$. The discrete Galerkin form for 42 can be obtained by following the same procedure as in Section 3. We only consider FEM, IGA and PUIGA implementations for solving this problem.

The cabin interior is studied in the frequency range 0.5 to $20 \mathrm{kHz}$ which is normally considered as the human audible frequency range. Frequencies toward the high end of the range, can impose a 

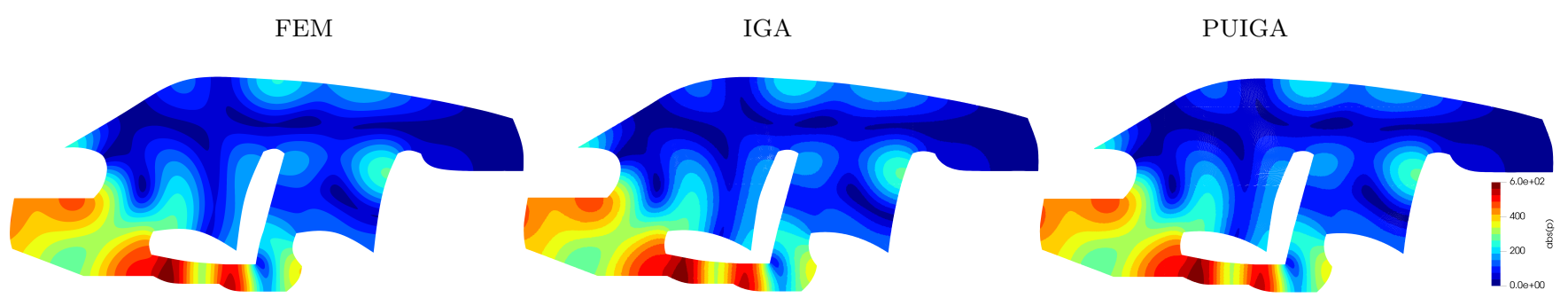

Figure 8: Car interior acoustics: Absolute value of the acoustic pressure in Pa inside the cabin for $0.5 \mathrm{kHz}$ where the converged solutions are obtained with FEM, IGA and PUIGA

significant computational cost due to the resulting short wavelengths. When performing the acoustic analyses for vehicle interiors, engineers need to construct several finite element meshes for the frequency range under consideration. Whether using FEM or IGA, it becomes necessary to refine the mesh grid in order to accommodate the higher frequencies i.e. the shorter wavelengths. Furthermore, the pollution error can significantly affect the accuracy at higher frequencies. This makes it necessary to increase the mesh resolution even further and therefore polynomial based methods may become computationally prohibitive. However, using plane wave enrichment it is possible to increase the number of degrees of freedom by adding more enrichment functions while retaining the same mesh. Removing the computational costs associated with the mesh refinement together with the improved efficiency makes enriched methods a more practical option for such problems. Finally, it should be noted that at short wavelengths numerical solutions become increasingly sensitive to small inaccuracies in representing the domain geometry. Hence, if a method is based on a geometry approximation it becomes necessary to improve the approximation at shorter wavelengths. This is often achieved by refining mesh grids. Again with PUIGA or IGA this can be avoided as the NURBS represent the exact geometry.

Starting with the lowest considered frequency i.e. $0.5 \mathrm{kHz}$ we first aim to test PUIGA $q$-refinement approach. The $q$-convergence of the method is achieved by retaining the same mesh and increasing the number of degrees of freedom. This convergence approach is already established for enriched methods in previous studies [12, 18, 46]. We compare the $q$-converged solution from PUIGA with a reference finite element solution that is achieved through the standard $h$-refinement approach. The reference solution is obtained by successively refining the mesh until the solution converges. The mesh considered for the reference solution, is shown in Figure6. Moreover, we also compare PUIGA and FEM solutions to an IGA solution achieved on a mesh composed of the same patches as PUIGA but with a much finer control mesh. Again IGA convergence is ensured by successively refining the knot vectors for each of the patch. For the comparison purpose we show in Figure 7 the considered meshes for IGA and PUIGA where the number of nodes and elements are indicated in the figure. We use second order interpolation in all the elements i.e. the bi-quadratic NURBS patches for IGA and PUIGA and the standard second order triangular elements for FEM. Furthermore, for PUIGA we solve the problem with 6,8 and 10 enrichment functions. The method converges at 8 enrichment functions. The converged solutions with different methods are displayed in Figure 8 . The figure shows that the acoustic pressure amplitude in Pa obtained with the three methods are qualitatively similar. To verify this further we performed several cross-sections on the data and compared the results at these sections. All the cross sections yielded similar results. For brevity sake we only include the plots shown in Figure 8.

To showcase the advantages of PUIGA we solve the problem again for higher frequencies while using the same coarse mesh as before. The $q$-converged solutions of the problem are displayed in Figure 9 for the frequencies $f=5,10,15$ and $20 \mathrm{kHz}$. The absolute magnitude of the acoustic 

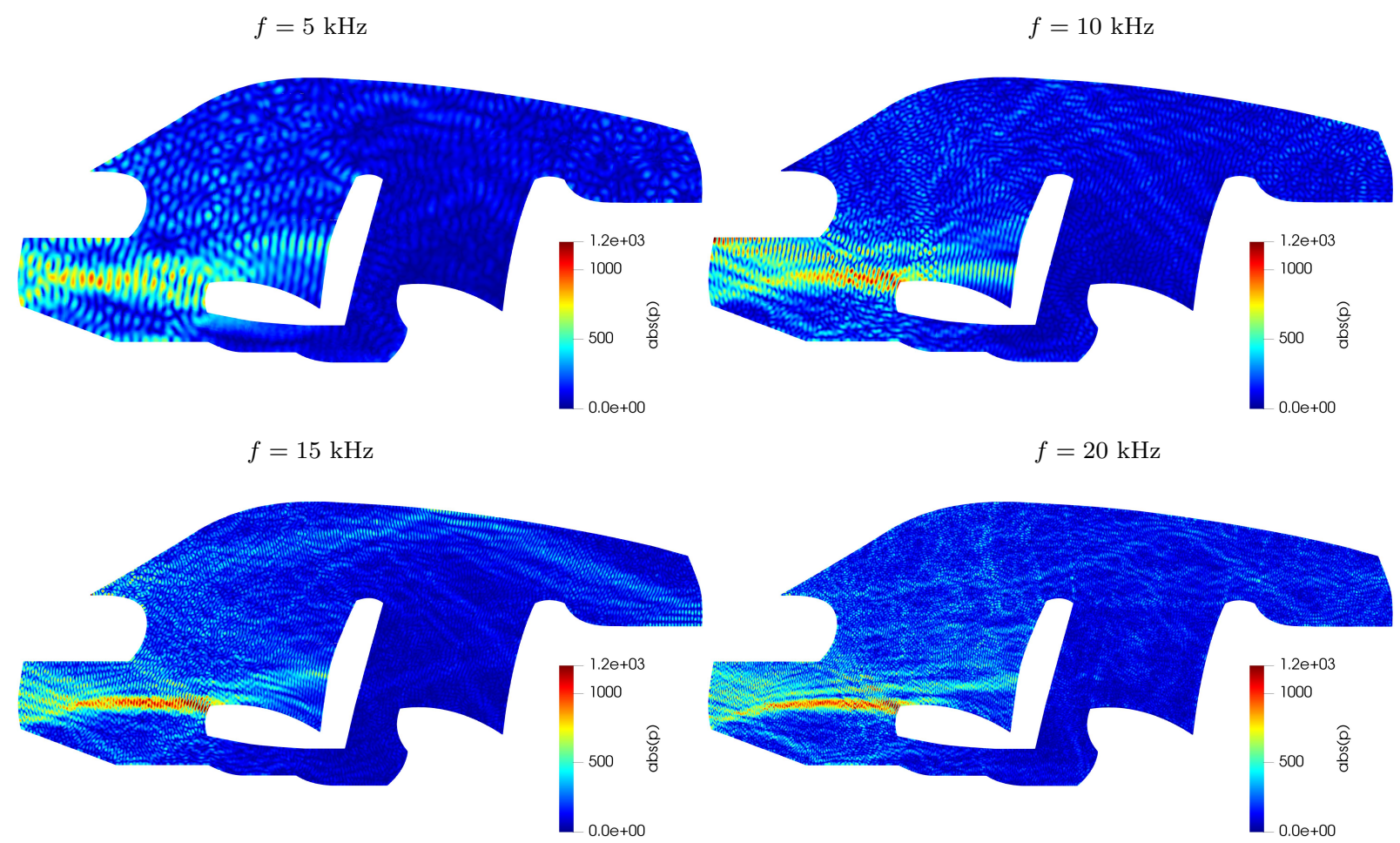

Figure 9: Car interior acoustics: Absolute value of the acoustic pressure in Pa inside the cabin for different frequencies obtained with PUIGA.

pressure of higher frequencies show a significant change in the interference patterns compared to the $0.5 \mathrm{kHz}$ case. The maximum constructive interferences can be noted under the driver seat in the latter case while in the former this can be observed between the source and the base of the driver seat. The impedance condition on the driver seat dampens the majority of higher frequency waves from arriving at the back seat while for the $0.5 \mathrm{kHz}$ frequency the waves are efficiently transmitted underneath the driver seat toward the back of the car cabin. Finally, for the $10 \mathrm{kHz}$ frequency a specific constructive interference seems to be forming near the top end of the boundary source. This might indicate specific resonant characteristics of the considered cabin.

\section{Conclusion}

The use of isogeometric analysis for wave problems is relatively new and the effect of higher continuity in the basis on the pollution error is still unclear. Our goal is to undertake a comparative computational investigation of FEM and IGA (and therefore PUFEM and PUIGA). The NURBS finite elements are compared to the Lagrangian finite elements for different polynomial orders. To quantify the pollution effect the solution errors are compared to the best approximation and empirical quasi-optimality constants are computed. The effect of using plane waves enriched basis functions is also taken into consideration where the pollution error for enriched isogeometric and Lagrangian elements are compared. The results suggest improved pollution behaviour with isogeometric analysis compared to Lagrangian elements where both enriched and non-enriched cases are considered. We have shown that although increasing the polynomial order in Lagrange FEM and PUFEM can handle the pollution, the quasi-optimality constant $C_{q}$ still grows with the wavenumber $k$. These observations are in-line with theory presented in [2]. However, this growth is observed to be either slower or roughly absent as $p$ grows for both IGA and PUIGA. 
The numerical examples show clear advantage for NURBS finite elements in term of pollution as well as efficiency where the same error can be achieved using fewer degrees of freedom. Based on this we propose using enriched isogeometric analysis for solving wave problems. Not only because of the improved pollution behaviour but also the exact geometry representation on coarse meshes. It was previously shown that inaccuracies in the geometry interpolation can significantly affect the finite element results for short wave problems. Hence, refined meshes become necessary even with enriched basis functions if Lagrangian polynomials are used to interpolate the geometry. This can be avoided if NURBS are used. Furthermore, adding enrichment to isogeometric analysis, eliminates the need for refining the mesh at higher frequencies where increasing the number of enrichment functions is enough. To showcase these advantages and to evaluate the enriched isogeometric analysis for geometries composed of multiple NURBS patches we study the noise patterns inside a car cabin at multiple frequencies. We simulate the car interior acoustics form $0.5 \mathrm{kHz}$ up to $20 \mathrm{kHz}$ using one coarse mesh.

\section{References}

[1] F. Ihlenburg and I. Babuška. Finite element solution of the Helmholtz equation with high wave number part I: The $h$-version of the FEM. Computers $\&$ Mathematics with Applications, 30(9):9-37, 1995.

[2] F. Ihlenburg. Finite element analysis of acoustic scattering, volume 132. Springer Science \& Business Media, 2006.

[3] M. Ainsworth. Discrete dispersion relation for $h p$-version finite element approximation at high wave number. SIAM Journal on Numerical Analysis, 42(2):553-575, 2004.

[4] M. Ainsworth, P. Monk, and W. Muniz. Dispersive and dissipative properties of discontinuous galerkin finite element methods for the second-order wave equation. Journal of Scientific Computing, 27(1-3):5-40, 2006.

[5] J. Melenk and S. Sauter. Convergence analysis for finite element discretizations of the Helmholtz equation with Dirichlet-to-Neumann boundary conditions. Mathematics of Computation, 79(272):1871-1914, 2010.

[6] J.M. Melenk and S. Sauter. Wavenumber explicit convergence analysis for Galerkin discretizations of the Helmholtz equation. SIAM Journal on Numerical Analysis, 49(3):1210-1243, 2011.

[7] F. Ihlenburg and I. Babuska. Finite element solution of the helmholtz equation with high wave number part ii: the hp version of the fem. SIAM Journal on Numerical Analysis, 34(1):315-358, 1997.

[8] J.M. Melenk and I. Babuška. The partition of unity finite element method: Basic theory and applications. Computer Methods in Applied Mechanics and Engineering, 139:289-314, 1996.

[9] I. Babuška and J.M. Melenk. The partition of unity method. International Journal for Numerical Methods in Engineering, 40:727-758, 1997.

[10] O. Laghrouche, P. Bettess, and R.J. Astley. Modelling of short wave diffraction problems using approximating systems of plane waves. International Journal for Numerical Methods in Engineering, 54:1501-1533, 2002. 
[11] O. Laghrouche, P. Bettess, E. Perrey-Debain, and J. Trevelyan. Plane wave basis finiteelements for wave scattering in three dimensions. International Journal for Numerical Methods in Biomedical Engineering, 19(9):715-723, 2003.

[12] A. El Kacimi and O. Laghrouche. Numerical modelling of elastic wave scattering in frequency domain by the partition of unity finite element method. International journal for numerical methods in engineering, 77(12):1646-1669, 2009.

[13] T. Strouboulis, I. Babuška, and R. Hidajat. The generalized finite element method for Helmholtz equation: Theory, computation, and open problems. Computer Methods in Applied Mechanics and Engineering, 195:4711-4731, 2006.

[14] T. Strouboulis and R. Hidajat. Partition of unity method for Helmholtz equation: q-convergence for plane-wave and wave-band local bases. Applications of Mathematics, 51(2):181-204, 2006.

[15] O. Laghrouche and M S. Mohamed. Locally enriched finite elements for the Helmholtz equation in two dimensions. Computers \& structures, 88:1469-1473, 2010.

[16] M S. Mohamed, A. El-Kacimi, and O. Laghrouche. Some numerical aspects of the PUFEM for efficient solution of 2D Helmholtz problems. Computers \& structures, 88:1484-1491, 2010.

[17] S. Banerjee and N. Sukumar. Exact integration scheme for planewave-enriched partition of unity finite element method to solve the Helmholtz problem. Computer Methods in Applied Mechanics and Engineering, 2017.

[18] T. Strouboulis, R. Hidajat, and I. Babuška. The generalized finite element method for Helmholtz equation part II: Effect of choice of handbook functions, error due to absorbing boundary conditions and its assessment. Computer Methods in Applied Mechanics and Engineering, 197:364$380,2008$.

[19] C. Farhat, I. Harari, and U. Hetmaniuk. A discontinuous Galerkin method with Lagrange multipliers for the solution of Helmholtz problems in the mid-frequency regime. Computer Methods in Applied Mechanics and Engineering, 192(11):1389-1419, 2003.

[20] R. Tezaur, L. Zhang, and C. Farhat. A discontinuous enrichment method for capturing evanescent waves in multiscale fluid and fluid/solid problems. Computer Methods in Applied Mechanics and Engineering, 197(19):1680-1698, 2008.

[21] R. Tezaur, I. Kalashnikova, and C. Farhat. The discontinuous enrichment method for mediumfrequency Helmholtz problems with a spatially variable wavenumber. Computer Methods in Applied Mechanics and Engineering, 268:126-140, 2014.

[22] T. Huttunen, M. Malinen, and P. Monk. Solving Maxwells equations using the ultra weak variational formulation. Journal of Computational Physics, 223(2):731-758, 2007.

[23] T. Luostari, T. Huttunen, and P. Monk. Improvements for the ultra weak variational formulation. International Journal for Numerical Methods in Engineering, 94(6):598-624, 2013.

[24] T. Luostari, T. Huttunen, and P. Monk. The ultra weak variational formulation of thin clamped plate problems. Journal of Computational Physics, 260:85-106, 2014. 
[25] N.C. Nguyen, J. Peraire, F. Reitich, and B. Cockburn. A phase-based hybridizable discontinuous Galerkin method for the numerical solution of the Helmholtz equation. Journal of Computational Physics, 290:318-335, 2015.

[26] H. Li, P. Ladevèze, and H. Riou. Hybrid finite element method and variational theory of complex rays for Helmholtz problems. Journal of Computational Acoustics, page 1650015, 2016.

[27] H. Li, P. Ladeveze, and H. Riou. Extended variational theory of complex rays in heterogeneous Helmholtz problem. Computational Mechanics, pages 1-10, 2017.

[28] R. Hiptmair, A. Moiola , and I. Perugia. A survey of Trefftz methods for the Helmholtz equation. In Building bridges: connections and challenges in modern approaches to numerical partial differential equations, pages 237-279. Springer, 2016.

[29] M. Dinachandra and S. Raju. Plane wave enriched partition of unity isogeometric analysis (PUIGA) for 2D-Helmholtz problems. Computer Methods in Applied Mechanics and Engineering, 335:380 - 402, 2018.

[30] M S. Mohamed, O. Laghrouche, and J. Trevelyan. A q-adaptive partition of unity finite element method for the solution of the 2-D Helmholtz equation. In IOP Conference Series: Materials Science and Engineering, volume 10, page 012148. IOP Publishing, 2010.

[31] T.JR Hughes, J.A Cottrell, and Y. Bazilevs. Isogeometric analysis: CAD, finite elements, NURBS, exact geometry and mesh refinement. Computer methods in applied mechanics and engineering, 194(39):4135-4195, 2005.

[32] Y. Bazilevs, V. M Calo, T.JR Hughes, and Y. Zhang. Isogeometric fluid-structure interaction: theory, algorithms, and computations. Computational mechanics, 43(1):3-37, 2008.

[33] C. Wang, M. CH Wu, F. Xu, M.C. Hsu, and Y. Bazilevs. Modeling of a hydraulic arresting gear using fluid-structure interaction and isogeometric analysis. Computers $\&$ Fluids, 142:3-14, 2017.

[34] A. Buffa, G. Sangalli, and R. Vázquez. Isogeometric analysis in electromagnetics: B-splines approximation. Computer Methods in Applied Mechanics and Engineering, 199(17):1143-1152, 2010 .

[35] A. Buffa and R. Vázquez. Isogeometric analysis for electromagnetic scattering problems. In Numerical Electromagnetic Modeling and Optimization for RF, Microwave, and Terahertz Applications (NEMO), 2014 International Conference on, pages 1-3. IEEE, 2014.

[36] J.A. Cottrell, A. Reali, Y. Bazilevs, and T.JR Hughes. Isogeometric analysis of structural vibrations. Computer methods in applied mechanics and engineering, 195(41):5257-5296, 2006.

[37] A. Cazzani, M. Malagù, E. Turco, and F. Stochino. Constitutive models for strongly curved beams in the frame of isogeometric analysis. Mathematics and Mechanics of Solids, 21(2):182209, 2016.

[38] T. Khajah, X. Antoine, and S. Bordas. Isogeometric finite element analysis of time-harmonic exterior acoustic scattering problems. arXiv preprint arXiv:1610.01694, 2016. 
[39] E. De Luycker, D.J. Benson, T. Belytschko, Y. Bazilevs, and M.C. Hsu. X-FEM in isogeometric analysis for linear fracture mechanics. International Journal for Numerical Methods in Engineering, 87(6):541-565, 2011.

[40] S. Sh Ghorashi, N. Valizadeh, S. Mohammadi, and T. Rabczuk. T-spline based XIGA for fracture analysis of orthotropic media. Computers \& structures, 147:138-146, 2015.

[41] V.P. Nguyen, C. Anitescu, S. Bordas, and T. Rabczuk. Isogeometric analysis: an overview and computer implementation aspects. Mathematics and Computers in Simulation, 117:89-116, 2015 .

[42] M.J. Peake, J. Trevelyan, and G. Coates. Extended isogeometric boundary element method (XIBEM) for two-dimensional Helmholtz problems. Computer Methods in Applied Mechanics and Engineering, 259:93-102, 2013.

[43] M.J. Peake, J. Trevelyan, and G. Coates. Extended isogeometric boundary element method (XIBEM) for three-dimensional medium-wave acoustic scattering problems. Computer Methods in Applied Mechanics and Engineering, 284:762-780, 2015.

[44] J.A. Cottrell, T.JR Hughes, and Y. Bazilevs. Isogeometric analysis: toward integration of CAD and FEA. John Wiley \& Sons, 2009.

[45] L. Coox, E. Deckers, D. Vandepitte, and W. Desmet. A performance study of NURBS-based isogeometric analysis for interior two-dimensional time-harmonic acoustics. Computer Methods in Applied Mechanics and Engineering, 305:441 - 467, 2016.

[46] G.C. Diwan, M S. Mohamed, M. Seaid, J. Trevelyan, and O. Laghrouche. Mixed enrichment for the finite element method in heterogeneous media. International Journal for Numerical Methods in Engineering, 101:54-78, 2015. 